\title{
Characterisation and the effects of bilirubin binding to human
}

\section{fibrinogen}

Nikola Gligorijević ${ }^{1 *}$, Simeon Minić ${ }^{2}$, Dragana Robajac ${ }^{1}$, Milan Nikolić ${ }^{2}$, Tanja Ćirković Veličković $c^{2,3,4,5}$, Olgica Nedić ${ }^{1}$

${ }^{1}$ Institute for the Application of Nuclear Energy, Department for Metabolism, University of Belgrade, Banatska 31b, 11080 Belgrade, Serbia

${ }^{2}$ Universtiy of Belgrade - Faculty of Chemistry, Department of Biochemistry and Centre of Excellence for Molecular Food Sciences, University of Belgrade, Studentski trg 12-16, 11000

Belgrade, Serbia

${ }^{3}$ Ghent University Global Campus, Yeonsu-gu, Incheon, South Korea

${ }^{4}$ Faculty of Bioscience Engineering, Ghent University, Ghent, Belgium

${ }^{5}$ Serbian Academy of Sciences and Arts, Knez. Mihailova 35, 11000 Belgrade, Serbia

*Correspondence to:

Nikola Gligorijević,

Institute for the Application of Nuclear Energy, Department for Metabolism

University of Belgrade

Banatska 31b, 11080 Belgrade, Serbia

Phone: +381113169058

E-mail: nikolag@inep.co.rs 


\section{ABSTRACT}

Fibrinogen, a protein involved in blood coagulation, is very susceptible to oxidation. Oxidation alters its function and usually makes it more thrombogenic. Bilirubin, an end-product of the haem degradation in vertebrates, is known for its antioxidant properties. The present paper describes interaction between fibrinogen and bilirubin, and the influence of bilirubin on the formation of fibrin and protection against oxidation. The binding constant of $4.5 \times 10^{4} \mathrm{M}^{-1}$ was determined for the fibrinogen/bilirubin complex at $37^{\circ} \mathrm{C}$. There is no change in secondary and tertiary structure of fibrinogen or its thermal stability upon bilirubin binding. The binding site of fibrinogen is not stereospecific for bilirubin and is able to accommodate both bilirubin conformers. A change in absorption maximum of bilirubin occurs upon its interaction with fibrinogen, suggesting an alteration in the conformation of bilirubin to the more cyclic one. Bilirubin exerts antioxidant effect on fibrinogen, preventing its carbonylation and aggregation. The presence of bilirubin induces the formation of fibrin with thicker fibres, as assessed by the coagulation assay. Fibrinogen and bilirubin interact at physiological concentrations, bilirubin may act as an antioxidant for fibrinogen and may modulate an important event in haemostasis, which altogether suggests possible physiological relevance of this interaction.

Keywords: Binding; Protein Oxidation; Coagulation; Fluorimetry; Circular Dichroism 


\section{INTRODUCTION}

Fibrinogen or factor I is a fibrillar protein with molecular mass of $340 \mathrm{kDa}$, composed of three pairs of chains forming $(\mathrm{A} \alpha \mathrm{B} \beta \gamma)_{2}$ structure [1]. Its main physiological role is in coagulation and it participates in both primary and secondary haemostasis. In primary haemostasis, fibrinogen enables platelet aggregation by the formation of bridges due to its interaction with IIIa/IIb glycoprotein [2]. In secondary haemostasis, fibrinogen is a precursor of the insoluble fibrin network which stabilises platelet clot [3]. In order to fulfil their roles, fibrinogen and fibrin interact with other proteins, ions and small molecules, which regulate the formation of fibrin and its degradation [4]. Fibrinogen interacts with tissue factors and cytokines, such as fibroblast growth factor-2, vascular endothelial growth factor and interleukin-1 $\beta$ [3]. Hyaluronic acid and S-nitrosoglutathione are able to bind to fibrinogen and fibrin causing modulation of their functions $[5,6]$.

Fibrinogen is very susceptible to oxidation [7], which significantly affects its function. Oxidatively modified fibrinogen, both in vivo and in vitro, becomes thrombogenic and more resistant to fibrinolysis compared to the unmodified molecule [8-11]. Fibrinogen undergoes glyco-oxidation in diabetes and related diseases causing formation of fibrin which is less efficient in the activation of plasmin and more resistant to hydrolysis [12]. There are, however, reports on the increased fibrinolysis and reduced clot strength caused by the oxidation of fibrinogen [13]. Therefore, fibrinogen oxidation, its consequences, mechanisms involved in the regulation and possible prevention of oxidation deserve further attention of the researchers and clinicians. 
Bilirubin, a tetrapyrrole pigment and a degradation product of the haem metabolism [14], has an antioxidant potential. Even very low, nanomolar concentrations of bilirubin in tissues may exert protective effect against oxidation in cooperation with biliverdin reductase in the biliverdinbilirubin cycle [15]. Taking into consideration the susceptibility of fibrinogen to oxidation and antioxidant properties of bilirubin, the interaction between these two molecules under physiological condition was investigated in this study, together with the consequences of bilirubin binding on the structure and stability of fibrinogen, fibrin formation and protection against oxidation.

\section{MATERIALS AND METHODS}

\subsection{Materials}

All substances used were purchased from Sigma-Aldrich (Germany). Fibrinogen was additionally purified: the commercial preparation was dissolved in $50 \mathrm{mM}$ phosphate buffer (PB) $\mathrm{pH} 7.3$, precipitated by the saturated ammonium sulphate solution (at the final concentration of $25 \%$ ), dissolved again in PB and dialysed against the same buffer at $4{ }^{\circ} \mathrm{C}$ overnight. The purity of fibrinogen was confirmed by the reducing SDS-PAGE (Supplementary Fig 1).

The concentration of fibrinogen (determined at $280 \mathrm{~nm}$ using the extinction coefficient of 15.1) in different experiments was different, and it was adjusted according to the analytical requirements of a specific method and the instrument. Bilirubin was dissolved in either DMSO or $10 \mathrm{mM} \mathrm{NaOH}$, but the final concentration of DMSO in reaction mixtures was below $1 \%$. All measurements, unless otherwise stated, were performed in duplicate immediately after mixing of the reactants.

\subsection{Spectrofluorometric analysis of the fibrinogen/bilirubin interaction}


The fluorescence data were obtained using FluoroMax®-4 spectrofluorometer (Horiba Scientific, Japan) at $37^{\circ} \mathrm{C}$. Fibrinogen $(20 \mathrm{nM})$ was enabled to interact with different amounts of bilirubin dissolved in DMSO $(0-20 \mu \mathrm{M})$. The excitation wavelength was chosen to be $280 \mathrm{~nm}$ (excitation of 41 tryptophan (Trp) and 67 tyrosine (Tyr) residues contributing to the intrinsic fluorescence of the protein) and the emission spectra were obtained in the range from 290 to 450 $\mathrm{nm}$, with the slits width of $5 \mathrm{~nm}$. Specific fluorescence obtained for each reaction pair was corrected for the background signal originating from the bilirubin itself. The differences in fluorescence intensities due to the inner-filter effect were corrected using the following equation [16]:

$\mathrm{Fc}=\mathrm{Fo} \times 10^{(\text {Aem+Aex }) / 2}(1)$

where $\mathrm{Fc}$ is the corrected fluorescence, Fo is the measured fluorescence, Aem and Aex correspond to the absorbance at the emission $(340 \mathrm{~nm})$ and the excitation $(280 \mathrm{~nm})$ peaks. The binding constant, $K_{\mathrm{a}}$, for the fibrinogen/bilirubin complex was calculated using the following equation:

$\log \frac{\mathrm{F}_{0}-\mathrm{F}}{\mathrm{F}}=-\operatorname{nlog} \frac{1}{[\mathrm{~L}]-[\mathrm{P}] \frac{\mathrm{F}_{0}-\mathrm{F}}{\mathrm{F}_{0}}}+\operatorname{nlogKa}(2)$

where $\mathrm{F}_{0}$ and $\mathrm{F}$ are the emission signals of the protein (fibrinogen) in the absence and in the presence of the ligand (bilirubin), while $[\mathrm{L}]$ and $[\mathrm{P}]$ correspond to the total concentrations of the ligand (bilirubin) and the protein (fibrinogen).

2.3 UV-VIS spectrometric analysis of the fibrinogen/bilirubin interaction

UV-VIS spectrometric characterisation of the fibrinogen/bilirubin complex was performed using ND-2000c microvolume spectrophotometer (Thermo Fisher Scientific, USA), in the range of 
$330-600 \mathrm{~nm}$ at room temperature. Spectra were recorded for fibrinogen $(4 \mu \mathrm{M})$, bilirubin $(36$ $\mu \mathrm{M})$ and their mixtures containing 2 or $4 \mu \mathrm{M}$ fibrinogen and $36 \mu \mathrm{M}$ bilirubin.

\subsection{Analysis of the thermal stability of the fibrinogen/bilirubin complex}

The influence of bilirubin on the thermal stability of fibrinogen was analysed using the same instrument as in section 2.2. Fibrinogen denaturation is characterised by a red shift of its emission peak at $340 \mathrm{~nm}$. The ratio between fluorescence intensities at 348 and $333 \mathrm{~nm}$ increases with temperature, enabling investigation of the thermal stability of fibrinogen. The temperature dependence of the fluorescence of fibrinogen, alone $(50 \mathrm{nM})$ or in the presence of bilirubin (20 $\mu \mathrm{M}$ ), was studied in the temperature range of 37 to $87^{\circ} \mathrm{C}$, with the rate of the temperature increase of $2{ }^{\circ} \mathrm{C} / \mathrm{min}$ (the equilibration period was set to $1 \mathrm{~min}$ ). The results were expressed as the change of the ratio $\mathrm{F}_{348} / \mathrm{F}_{333}$ with the temperature and presented as a sigmoidal function. The inflection point in the plot was considered as the melting temperature (Tm) of fibrinogen.

\subsection{CD spectrometric analysis of the fibrinogen/bilirubin interaction}

$\mathrm{CD}$ spectrometric characterisation of the fibrinogen/bilirubin complex was performed using J815 spectropolarimeter (Jasco, Japan), at $37^{\circ} \mathrm{C}$ and a scan speed of $50 \mathrm{~nm} / \mathrm{min}$. The near-UV measurements were performed in the range of $250-350 \mathrm{~nm}$ with the cell path of $1 \mathrm{~cm}$. The concentration of fibrinogen was kept constant $(4 \mu \mathrm{M})$ while the concentration of bilirubin was varied $(18,36$ and $54 \mu \mathrm{M})$. For the far-UV measurements $(185-260 \mathrm{~nm}, 0.1 \mathrm{~cm}$ cell path), the same concentrations of fibrinogen and bilirubin were tested, however this time bilirubin was dissolved in $10 \mathrm{mM} \mathrm{NaOH}$, as DMSO interferes at these wavelengths.

2.6 Investigation of the protective effect of bilirubin against oxidation of fibrinogen 
In this experiment, 2,2'-azobis(2-amidinopropane) dihydrochloride (AAPH) was used as an oxidising reagent. The oxidation of fibrinogen $(3 \mu \mathrm{M}, 1 \mathrm{~mL})$ was performed using AAPH (at the final concentration of $5 \mathrm{mM})$ in the absence and in the presence of bilirubin $(6 \mu \mathrm{M}$ dissolved in $10 \mathrm{mM} \mathrm{NaOH})$. The reaction mixture was incubated at $37^{\circ} \mathrm{C}$ and $200 \mu \mathrm{L}$ aliquots were taken at defined time intervals (Fig 4). An ice-cold $20 \%$ TCA $(200 \mu \mathrm{L})$ was added to each aliquot and the proteins precipitated by centrifugation at $12000 \mathrm{x} g$ for $20 \mathrm{~min}$. The obtained pellet was washed with an ice-cold acetone $(400 \mu \mathrm{L})$, centrifuged again and dissolved in the sample buffer for the non-reducing PAGE. The proteins were resolved by the native PAGE using $8 \%$ gel and transferred on the poly(vinylidene fluoride) membrane (PVDF). Protein carbonyls were derivatised in situ using dinitrophenylhydrazine (DNP) and immunoblotted using anti-DNP antibody $[17,18]$.

\subsection{Investigation of the effect of bilirubin binding to fibrinogen on its coagulation}

A coagulation assay, i.e. the formation of fibrin from fibrinogen, was conducted in a microtiter plate using Victor $^{3} \mathrm{~V}$ multilabel reader (PerkinElmer, USA) at the room temperature. The coagulation of fibrinogen $(3 \mu \mathrm{M})$ was performed using $\mathrm{CaCl}_{2}$ (at the final concentration of 2.2 $\mathrm{mM})$ and thrombin $(1 \mathrm{IU} / \mathrm{mL})$ in the absence and in the presence of bilirubin $(6 \mu \mathrm{M}$ dissolved in $10 \mathrm{mM} \mathrm{NaOH})$. The absorbance of the mixture containing all reactants except fibrinogen was also recorded and used to correct the corresponding result obtained in the presence of fibrinogen. The preliminary testing confirmed that $10 \mathrm{mM} \mathrm{NaOH}$ had very little effect on the formation of fibrin. The coagulation process was monitored at $350 \mathrm{~nm}$, at $10 \mathrm{sec}$ intervals for $8 \mathrm{~min}$. All measurements were performed in triplicate. 


\section{RESULTS}

\subsection{Spectrometric analysis of the fibrinogen/bilirubin interaction}

The interaction between bilirubin and fibrinogen caused the concentration-dependent reduction of the intrinsic fluorescence of fibrinogen (Fig 1A). Using the quenching data, a binding constant for the fibrinogen/bilirubin complex was determined to be $4.5 \times 10^{4} \mathrm{M}^{-1}$ at $37^{\circ} \mathrm{C}$ (Fig 1B). No shift in the emission spectra maximum was observed as a consequence of the bilirubin binding, suggesting that amino acids Tyr and Trp in fibrinogen remain in the same or very similar environment in the native and bilirubin-bound protein. UV-VIS spectrometry confirmed the existence of the fibrinogen/bilirubin interaction. The absorption maximum of bilirubin became wider and a red shift was observed in the presence of either 2 or $4 \mu \mathrm{M}$ fibrinogen, although it was more pronounced in the presence of the higher concentration of the protein (Fig 1C).

\subsection{Analysis of the thermal stability of the fibrinogen/bilirubin complex}

As explained in the section 2.4, thermal denaturation of fibrinogen induces a red shift of its emission peak. Experiments with fibrinogen and fibrinogen/bilirubin complex have shown no difference in their thermal stability, i.e. the melting points were calculated to be $54{ }^{\circ} \mathrm{C}$ in both cases (Fig 2). This result indicated that bilirubin binding does not affect fibrinogen stability. 3.3 CD spectrometric analysis of the fibrinogen/bilirubin interaction Results obtained using CD-spectrometry confirmed the previously obtained data. Near-UV CD spectra (Fig 3A) and far-UV CD spectra (Fig 3B) confirmed that bilirubin binding to fibrinogen does not affect its tertiary or secondary structure. The far-UV CD spectrum, which results from the absorption originating from the $\alpha$-helix secondary structure of fibrinogen remained almost unchanged upon its interaction with bilirubin. 


\subsection{Investigation of the protective effect of bilirubin on the oxidation of fibrinogen}

Incubation of fibrinogen with AAPH induced oxidation which resulted in a partial protein aggregation (Fig 4A). The addition of bilirubin reduced a degree of fibrinogen modification during the first hour of the oxidative attack, but after two hours bilirubin was much less efficient in preventing protein aggregation (Fig 4A). Derivatization of the protein carbonyls (i.e. oxidative modifications) with DNP and their subsequent detection with anti-DNP antibody confirmed the existence of the lower amount of fibrinogen carbonyl groups in the presence of bilirubin (Fig 4B and 4C). Another indicator of the bilirubin interaction was the colour of the solution - after two hours of incubation bilirubin lost its yellow colour.

\subsection{Investigation of the effect of bilirubin binding to fibrinogen on its coagulation}

A coagulation assay demonstrated that bilirubin binding to fibrinogen affected the formation of fibrin (Fig 5). The initial speed of fibrin formation and the coagulation times for fibrinogen and fibrinogen/bilirubin complex were very similar, whereas the maximal absorbance of the fibrin formed in the presence of bilirubin was much higher than the absorbance of the fibrin formed from fibrinogen alone. This finding implicated that thicker fibrin fibres form in the presence of bilirubin.

\section{DISCUSSION}

A newly discovered interaction between fibrinogen and bilirubin is described in this paper. Bilirubin in blood, at the concentration up to $30 \mu \mathrm{M}$ in healthy persons [19], is predominantly bound to albumin with a high affinity constant that ranges from $6.7 \times 10^{6}$ to $>10^{8} \mathrm{M}^{-1}$. The binding constant is concentration- and temperature-dependent [20]. The affinity of bilirubin for 
fibrinogen is much lower and is calculated to be $4.5 \times 10^{4} \mathrm{M}^{-1}$. According to our results, bilirubin binding to fibrinogen does not influence the structure or the melting point of fibrinogen [21]. Phycocyanobilin, a molecule with the structure similar to bilirubin, binds to and stabilises human serum albumin (HSA) by increasing its $\alpha$-helical content and the melting point [22]. In aqueous solution, bilirubin exists in an equimolar ratio of $M$ and $P$ ridge tile conformers. It is known that stereoselective binding of the $P$ conformer to HSA induces optical activity, i.e. a positive Cotton effect in the CD spectra of the HSA/bilirubin complex [23]. According to our results (data not shown), the formation of fibrinogen/bilirubin complex is not accompanied by a stereoselective binding of this pigment. Therefore, the binding site of fibrinogen is not stereospecific for bilirubin and is able to accommodate both conformers.

When added to a solution of fibrinogen, a red shift of the absorption maximum of bilirubin was observed together with the change in the shape of the peak, suggesting an alteration in the conformation of bilirubin upon its binding to fibrinogen. Spectrometric calculations predict that a transition of bilirubin from a ridge tile to a more cyclic conformation is accompanied by a red shift of its absorption maximum [23]. Therefore, the results presented in this work suggest that bilirubin bound to fibrinogen probably exists in a more cyclic conformation. A similar shift was observed upon bilirubin binding to HSA [24-26]. In contrast to the HSA/bilirubin complex, which was characterised by a large increase in the fluorescence of bilirubin [27], this phenomenon was not observed in the case of the fibrinogen/bilirubin complex (data not shown). Since the fluorescence correlates with a degree of structural rigidness and the binding of bilirubin to fibrinogen is much weaker than to HSA, it can be postulated that the binding to fibrinogen does not affect a conformational freedom of bilirubin significantly and, hence, its fluorescence. 
Certain pathophysiological conditions are characterised by an increased oxidative stress. Oxidation alters the structure and function of proteins [28]. Fibrinogen is very susceptible to oxidation which usually makes it more thrombogenic. An increased oxidation of fibrinogen was detected in patients with myocardial infarction, cirrhosis or diabetes mellitus [10,12,29]. An oxidative stress affects the entire coagulation system causing an increase in the reactivity of platelets and a dysfunction of the endothelium [30]. In order to investigate a possible protective role of bilirubin binding against the oxidation of fibrinogen, reactants were mixed at their physiological concentrations and ratios. An antioxidant effect of bilirubin was clearly demonstrated, as the presence of bilirubin prevented carbonylation and aggregation of fibrinogen.

Beneficial effects of bilirubin on the human health were reported in several studies. A negative correlation was found between its concentration and an incidence of the colorectal cancer [31] or prediabetes [32]. In the patients with diabetes and Gilbert syndrome, whose bilirubin concentrations may reach up to $90 \mu \mathrm{M}$ [33], a decrease in the oxidation of proteins was recorded [34]. Bilirubin was recognised as an endogenous antioxidant in human endothelial cells [35] and its concentration is negatively correlated with the concentration of fibrinogen and plasminogen activator inhibitor-1 [36].

In order to investigate a potential effect of bilirubin bound to fibrinogen, a coagulation assay was performed (again, using physiological concentrations and ratios of fibrinogen and bilirubin). The presence of bilirubin induced formation of thicker fibrin fibres, affecting its branching and permeability, since it is known that thinner fibres result in higher branching and reduced permeability $[10,37]$. When permeability is reduced, plasmin cannot efficiently penetrate in the 
clot, leading to a prolonged degradation time of fibrin and an increased risk of the formation of thrombus [28]. The effect of bilirubin on the formation of fibrin was the same as the effect of the aspirin treatment [38]. The data obtained in our study point to bilirubin as a beneficial modulator of haemostasis and a protector of fibrinogen against oxidation. Although fibrin with thicker fibres usually has higher permeability, other factors may influence the final outcome $[11,39]$. One should keep in mind that the coagulation assay employed in this work was based on the purified fibrinogen and a commercially available thrombin. Many other factors present in blood influence the formation of fibrin [4]. Nevertheless, fibrinogen and bilirubin interact at physiological concentrations, bilirubin may protect fibrinogen against oxidation and may modulate fibrin formation, which altogether suggests a possible physiological relevance of this interaction.

\section{Acknowledgements}

This work was supported by the Ministry of Education, Science and Technological Development of Serbia [grant numbers 173042 and 172024].

\section{REFERENCES}

[1] S.T. Lord, Molecular mechanisms affecting fibrin structure and stability, Arterioscler. Thromb. Vasc. Biol. 31 (2011) 494-499. https://doi.org/10.1161/ATVBAHA.110.213389. [2] J.S. Bennett, Platelet-fibrinogen interactions, Ann. N. Y. Acad. Sci. 936 (2001) 340-354. https://doi.org/10.1111/j.1749-6632.2001.tb03521.x. 
[3] M.W. Mosesson, Fibrinogen and fibrin structure and functions, J. Thromb. Haemost. 3

(2005) 1894-1904. https://doi.org/10.1111/j.1538-7836.2005.01365.x.

[4] J.W. Weisel, R.I. Litvinov, Fibrin formation, structure and properties, Subcell. Biochem. 82 (2017) 405-456. https://doi.org/10.1007/978-3-319-49674-0_13.

[5] R.D. LeBoeuf, R.H. Raja, G.M. Fuller, P.H. Weigel, Human fibrinogen specifically binds hyaluronic acid, J. Biol. Chem. 261 (1986) 12586-12592.

[6] R.M. Bateman, C.G. Ellis, M. Suematsu, K.R. Walley, S-nitrosoglutathione acts as a small molecule modulator of human fibrin clot architecture, PLoS One. 7 (2012) e43660. https://doi.org/10.1371/journal.pone.0043660.

[7] E. Shacter, J.A. Williams, M. Lim, R.L. Levine, Differential susceptibility of plasma proteins to oxidative modification: examination by western blot immunoassay, Free Rad. Biol. Med. 17 (1994) 429-437. https://doi.org/10.1016/0891-5849(94)90169-4.

[8] G.R. Upchurch Jr, N. Ramdev, M.T. Walsh, J. Loscalzo, Prothrombotic consequences of the oxidation of fibrinogen and their inhibition by aspirin, J. Thromb. Thrombolysis. 5 (1998) 9-14. https://doi.org/10.1023/A:1008859729045.

[9] G. Lupidi, M. Angeletti, A.M. Eleuteri, L. Tacconi, M. Coletta, E. Fioretti, Peroxynitritemediated oxidation of fibrinogen inhibits clot formation, FEBS Lett. 462 (1999) 236-240. https://doi.org/10.1016/S0014-5793(99)01500-8.

[10] M. Becatti, R. Marcucci, G. Bruschi, N. Taddei, D. Bani, A.M. Gori, B. Giusti, G.F. Gensini, R. Abbate, C. Fiorillo, Oxidative modification of fibrinogen is associated with altered function and structure in the subacute phase of myocardial infarction, Arterioscler. Thromb. Vasc. Biol. 34 (2014) 1355-1361. https://doi.org/10.1161./ATVBAHA.114.303785. 
[11] G.C.G. Hugenholtz, F. Macrae, J. Adelmeijer, S. Dulfer, R.J. Porte, T. Lisman, R.A.S. Ariëns, Procoagulant changes in fibrin clot structure in patients with cirrhosis are associated with oxidative modifications of fibrinogen, J. Thromb. Haemost. 14 (2016) 1054-1066. https://doi.org/10.1111/jth.13278.

[12] E.J. Dunn, H. Philippou, R.A. Ariëns, P.J. Grant, Molecular mechanisms involved in the resistance of fibrin to clot lysis by plasmin in subjects with type 2 diabetes mellitus, Diabetologia. 49 (2006) 1071-1080. https://doi.org/10.1007/s00125-006-0197-4.

[13] N.J. White, Y. Wang, X. Fu, J.C. Cardenas, E.J. Martin, D.F. Brophy, C.E. Wade, X. Wang, A.E. St. John, E.B. Lim, S.A. Stern, K.R. Ward, J.A. López, D. Chung, Post-translational oxidative modification of fibrinogen is associated with coagulopathy after traumatic injury, Free Rad. Biol. Med. 96 (2016) 181-189. https://doi.org/10.1016/j.freeradbiomed.2016.04.023. [14] X. Wang, J.R. Chowdhury, N.R. Chowdhury, Bilirubin metabolism: applied physiology, Curr. Paediatr. 16 (2006) 70-74. https://doi.org/10.1016/j.cupe.2005.10.002.

[15] T.W. Sedlak, M. Saleh, D.S. Higginson, B.D. Paul, K.R. Juluri, S.H. Snyder, Bilirubin and glutathione have complementary antioxidant and cytoprotective roles, Proc. Natl. Acad. Sci. USA. 31 (2009) 5171-5176. https://doi.org/10.1073/pnas.0813132106.

[16] J.R. Lakowicz, Principles of fluorescence spectroscopy, Third ed., Springer, Boston, 2006. [17] C.E. Robinson, A. Keshavarzian, D.S. Pasco, T.O. Frommel, D.H. Winship, E.W. Holmes, Determination of protein carbonyl groups by immunoblotting, Anal. Biochem. 266 (1999) 48-57. https://doi.org/10.1006/abio.1998.2932. 
[18] N. Gligorijević, S. Minić, M. Križáková, J. Katrlík, O. Nedić, Structural changes of fibrinogen as a consequence of cirrhosis, Thromb. Res. 166 (2018) 43-49. https://doi.org/10.1016/j.thromres.2018.04.005.

[19] M.K Kringen, A.P. Piehler, R.M. Grimholt, M.S. Opdal, K.B.F. Haug, P. Urdal, Serum bilirubin concentration in healthy adult north-Europeans is strictly controlled by the UGT1A1 TA-repeat variants, PLoS One. 9 (2014), e90248. https://doi.org/10.1371/journal.pone.0090248. [20] L. Roca, S. Calligaris, R.P. Wennberg, C.E. Ahlfors, S.G. Malik, J.D. Ostrow, C. Tiribelli, Factors affecting the binding of bilirubin to serum albumins: validation and application of the peroxidase method, Pediatr. Res. 60 (2006) 724-728. https://doi.org/ 10.1203/01.pdr.0000245992.89965.94.

[21] N. Hassan, L.R. Barbosa, R. Itri, J.M. Ruso, Fibrinogen stability under surfactant interaction, J. Colloid Interface Sci. 362 (2011) 118-126.

https://doi.org/10.1016/j.jcis.2011.06.010.

[22] M. Radibratovic, S. Minic, D. Stanic-Vucinic, M. Nikolic, M. Milcic, T. Cirkovic Velickovic., Stabilization of human serum albumin by the binding of phycocyanobilin, a bioactive chromophore of blue-green alga Spirulina: molecular dynamics and experimental study, PLoS One. 11 (2016) e0167973. https://doi.org/10.1371/journal.pone.0167973. [23] C.E. Petersen, C-E. Ha, K. Harohalli, J.B. Feix, N.V. Bhagavan, A dynamic model for bilirubin binding to human serum albumin, J. Biol. Chem. 275 (2000) 20985-20995. https://doi.org/10.1074/jbc.M001038200. 
[24] J. Jasprova, M.D. Ben, E. Vianello, I. Goncharova., M. Urbanova, K. Vyroubalova, S. Gazzin, C. Tiribelli, M. Sticha, M. Cerna, L. Vitek, The biological effects of bilirubin photoisomers, PLoS One. 11 (2016) e0148126. https://doi.org/10.1371/journal.pone.0148126. [25] J. Lou, K.S. Birdi, Interaction of bilirubin with human serum albumin and cationic detergents, Z. Naturforsch. C. 28 (1973) 511-513.

[26] G. Blauer, T.E. King, Interactions of bilirubin with bovine serum albumin in aqueous solution, J. Biol. Chem. 245 (1970) 372-381.

[27] G.H. Beaven, A. d'Albis, W.B. Gratzer, The interaction of bilirubin with human serum albumin, Eur. J. Biochem. 33 (1973) 500-517. https://doi.org/ 10.1111/j.1432-1033.1973.tb02709.x. [28] J.M. Curtis, W.S. Hahn, E.K. Long, J.S. Burrill, E.A. Arriaga, D.A. Bernlohr, Protein carbonylation and metabolic control systems, Trends Endocrinol. Metab. 23 (2012) 399-406. https://doi.org/10.1016/j.tem.2012.05.008.

[29] T. Lisman, R.A.S. Ariëns, Alterations in fibrin structure in patients with liver diseases, Semin. Thromb. Hemost. 42 (2016) 389-396. https://doi.org/ 10.1055/s-0036-1572327. [30] N. Kakouros, J.J. Rade, A. Kourliouros, J.R. Resar, Platelet function in patients with diabetes mellitus: from a theoretical to a practical perspective. Int. J. Endocinol. 2011, Article ID 742719. https://doi.org/10.1155/2011/742719.

[31] S.D. Zucker, P.S. Horn, K.E. Sherman, Serum bilirubin levels in the U.S. population: effect and inverse correlation with colorectal gender cancer, Hepatology 40 (2004) 827-835. https://doi.org/10.1002/hep.20407. 
[32] M. Bossard, S. Aeschbacher, T. Schoen, T. Hochgruber, M. von Rotz, J. Blum, M. Risch, L. Risch, D. Conen, Serum bilirubin levels and risk of prediabetes in young and healthy adults, Int. J. Cardiol. 171 (2014) e24-e25. https://doi.org/10.1016/j.ijcard.2013.11.125.

[33] P.J. Bosma, J.R. Chowdhury, C. Bakker, S. Gantla, A. de Boer, B.A. Oostra, D. Lindhout, G.N.J. Tytgat. P.L.M. Jansen, R.P.J.O. Elferink, N.R. Chowdhury, The genetic basis of the reduced expression of bilirubin UDP-glucuronosyltransferase 1 in Gilbert's syndrome, New Engl. J. Med. 333 (1995) 1171-1175. https://doi.org/10.1056/NEJM199511023331802.

[34] T. Maruhashi, J. Soga, N. Fujimura, N. Idei, S. Mikami, Y. Iwamoto, M. Kajikawa, T. Matsumoto, Y. Kihara, K. Chayama, K. Noma, A. Nakashima, H. Tomiyama, B. Takase, A. Yamashina, Y. Higashi, Hyperbilirubinemia, augmentation of endothelial function, and decrease in oxidative stress in Gilbert syndrome, Circulation 126 (2012) 598-603. https://doi.org/10.1161/CIRCULATIONAHA.112.105775.

[35] L. Ziberna, M. Martelanc, M. Franko, S. Passamonti, Bilirubin is an endogenous antioxidant in human vascular endothelial cells. Sci. Rep. 6 (2016) 29240.

https://doi.org/10.1038/srep29240.

[36] H.S. Cho, S.W. Lee, E.S. Kim, J. Shin, S.D. Moon, J.H. Han, B.Y. Cha, Serum bilirubin levels are inversely associated with PAI-1 and fibrinogen in Korean subjects, Atherosclerosis, 244 (2016) 204-210. https://doi.org/10.1016/j.atherosclerosis.2015.11.008.

[37] E.M. Scott, R.A.S. Ariëns, P.J. Grant, Genetic and environmental determinants of fibrin structure and function, relevance to clinical disease, Arterioscler. Thromb. Vasc. Biol. 24 (2004) 1558-1566. https://doi.org/10.1161/01.ATV.0000136649.83297.bf. 
[38] R.A. Ajjan, K.F. Standeven, M. Khanbhai, F. Phoenix, K.C. Gersh, J.W. Weisel, M.T. Kearney, R.A.S. Ariëns, P.J. Grant, Effects of aspirin on clot structure and fibrinolysis using a novel in vitro cellular system, Arterioscler. Thromb. Vasc. Biol. 29 (2009) 712-717. https://doi.org/10.1161/ATVBAHA.109.183707.

[39] A.R. Wufsus, N.E. Macera, K.B. Neeves, The hydraulic permeability of blood clots as a function of fibrin and platelet density, Biophys. J. 104 (2013) 1812-1823.

https://doi.org/10.1016/j.bpj.2013.02.055. 


\section{Figure legends}

Fig 1. The emission spectra of fibrinogen in the absence and in the presence of different concentrations of bilirubin (A). Determination of the binding constant of the fibrinogen/bilirubin complex (B). UV-VIS spectra of bilirubin, fibrinogen and bilirubin/fibrinogen complex (C).

Fig 2. Determination of the melting point of fibrinogen and the fibrinogen/bilirubin complex; the ratio of fluorescence intensities at 348 and $333 \mathrm{~nm}\left(\mathrm{~F}_{348} / \mathrm{F}_{333}\right)$ was calculated. The inflection point on the curve corresponds to the melting point.

Fig 3. Near-UV (A) and far-UV (B) CD spectra of fibrinogen in the absence and in the presence of bilirubin.

Fig 4. Determination of the susceptibility of fibrinogen to oxidation with AAPH in the absence () and in the presence (+) of bilirubin during $4 \mathrm{~h}$. A native PAGE and a transfer to the PVDF membrane was performed, followed by the protein staining with Ponceau S (A), and derivatization of the protein carbonyls with DNP and immunoblotting with anti-DNP antibody (B). The ratio of densitometric signals obtained by immunoblotting and Ponceau S staining (C) (there was no protein detected by Ponceau S staining after $240 \mathrm{~min}$, although an immunoreactive signal with anti-DNP antibody was recorded).

Fig 5. Determination of the effect of bilirubin on the formation of fibrin in the coagulation assay. 

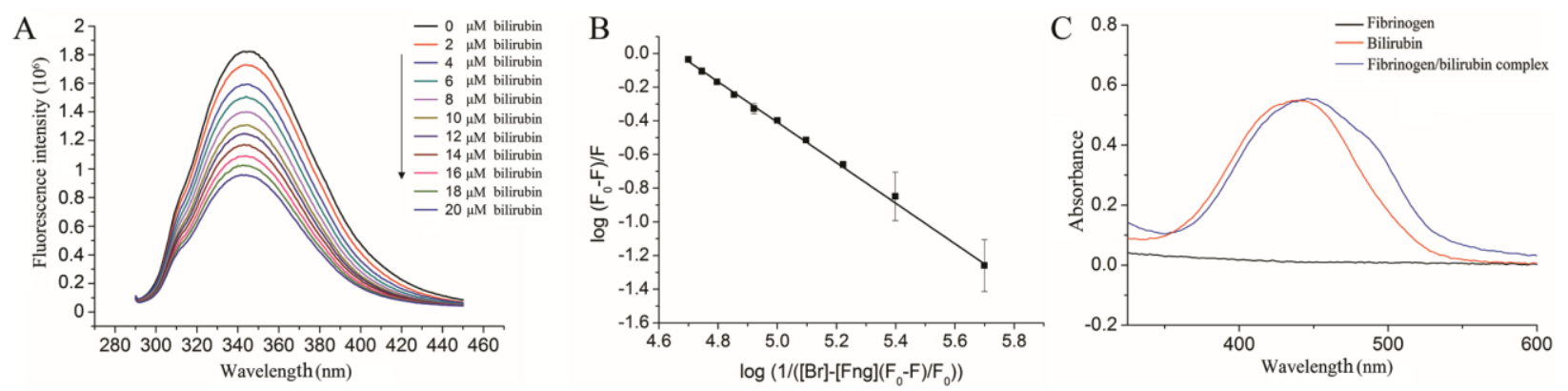

Figure 1

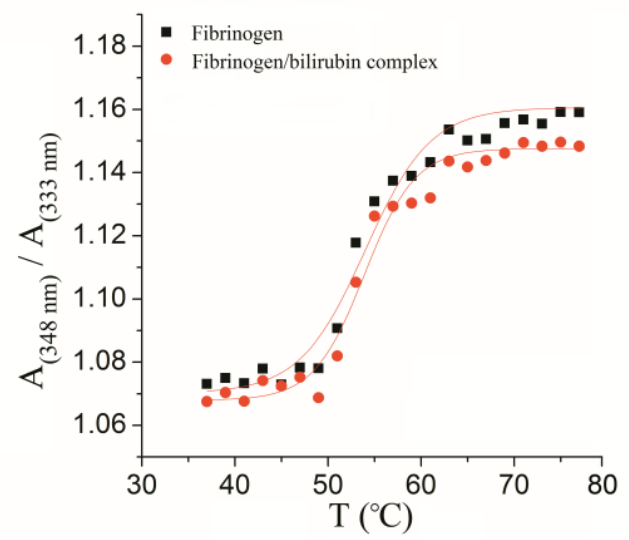

Figure 2
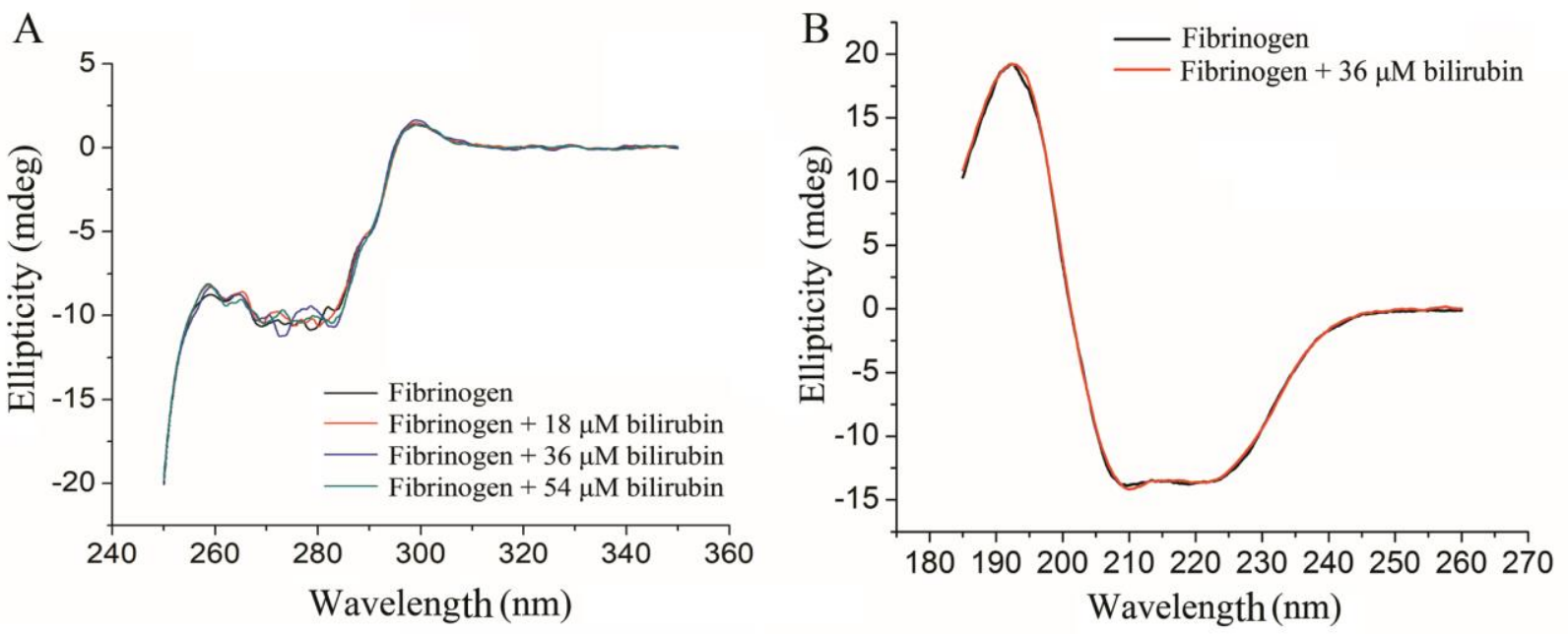

Figure 3 


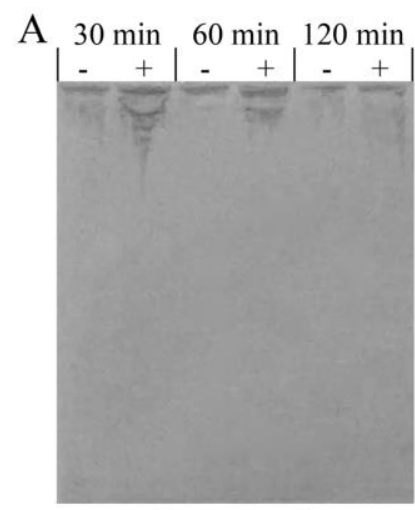

Ponceau S

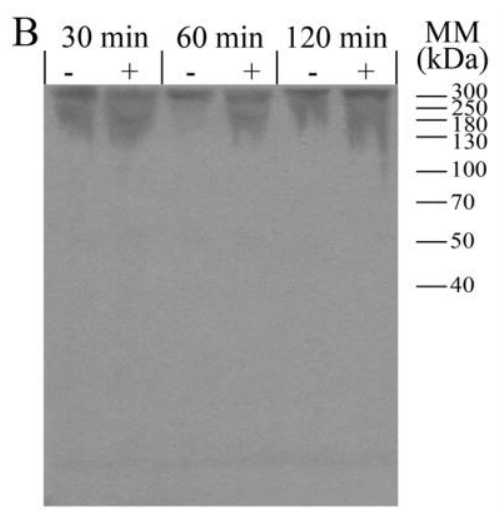

Anti-DNP

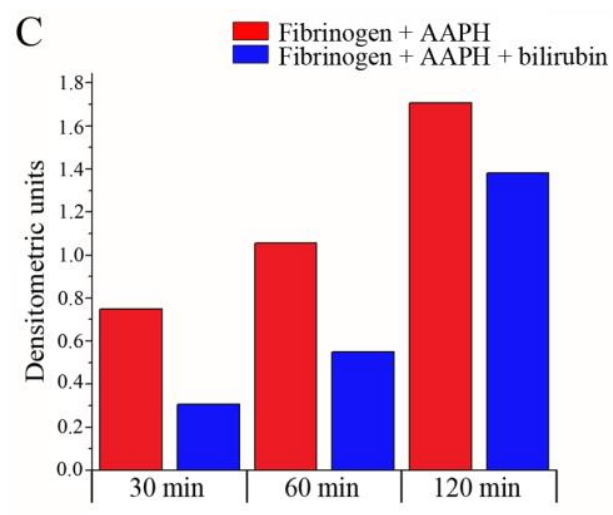

Figure 4

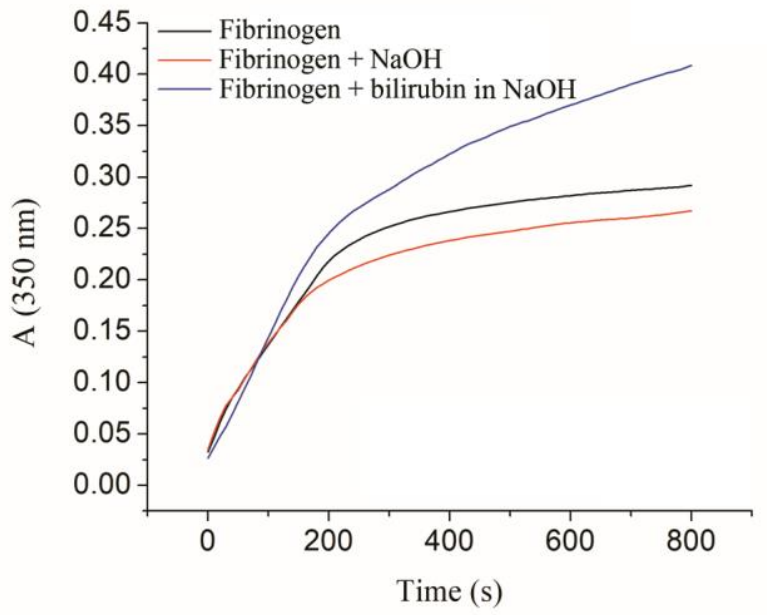

Figure 5 


\section{Supplementary figure}

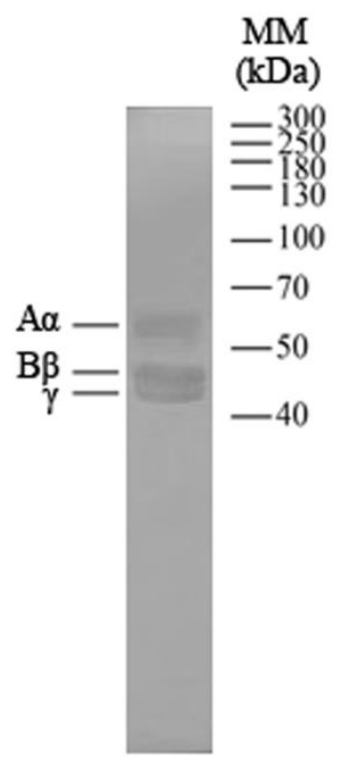

Supplementary Fig.1. Ponceau S staining of the purified fibrinogen purchased from SigmaAldrich after reducing SDS-PAGE and transfer to PVDF membrane. 